\title{
An Automated Pelvic Bone Geometrical Feature Measurement Utilities on Ct Scanning
}

\author{
Dr. Shubangi D.C. ${ }^{1}$, B. Samirana Acharya ${ }^{2}$ \\ ${ }^{I}$ Professor, Department of computer science and engineering, Poojya doddappa Appa College of engineering, \\ Gulbarga, Karnataka, India, \\ ${ }^{2}$ Department of computer science and engineering, Poojya doddappa Appa College of engineering, Gulbarga,
} Karnataka, India,

\begin{abstract}
An accurate depth location of pelvic land mark could be affected by the acoustic properties of the penetrated tissues and improper depth estimation could lead to a miscalculation of the acetabular cup implant. In the case of obese patient it is very difficult to locate the place of damage. Errors in land mark location leads to the improper treatment by orthopedic surgeons. The aim of this system is to determine pelvic orientation from CT scan images based on patch statistical shape model multilevel measurement utility tools by image calibration system; by applying a hierarchical registration scheme. Here a plastic bones and cadaveric specimen are used. Many approaches have been proposed in the literature to replace the percutanesous digitization, Involvement of the experts in the system to give the solution or conclusion.

Key words CT-Scanning, Patch statistical shape model (SSM), pelvis.
\end{abstract}

Submitted Date 24 June 2013

Accepted Date: 29 June 2013

\section{Introduction}

The pelvis, or pelvic bone, derived from the Latin word for basin is an anatomical structure found in most vertebrates. It is the name given to both a bony structure and pelvic girdle connecting the base of the spine to the rear limbs, and the region of the body defined by that structure .The muscles and tissue beneath the pelvic girdle are known as the pelvic floor. The rounded epiphysis of the femur called the Head articulates with the pelvic bone at the Hip Joint at the acetabulum. In the misaligned cup prosthesis are generally reduced range of motion increased wear and dislocations [1]-[4].Therefore, the effect on the orientation of the acetabular cup has been the focus research for long time [5]-[7]. For the last 10-15 years various concepts have been introduced to measure the orientation of the acetabular component. Recent inventions like mechanical guidance instruments [8], [9], many computer assisted strategies have been developed and successfully applied to THA's. The image based strategies either relay an preoperative CT scans [10]-[11] or on intra operative images fluoroscopy [12][13].And these strategies increased operative time and further both patient and surgical staff are exposed to radiations imageless strategies have developed. The APP is a common reference plane and derived by three land marks. The bilateral ASIS and the midpoint between the two pubercles [20]-[21], this land mark identification step is normally done using a tracked pointer. However even a minor failure in correct landmark identification could lead to an increased error of cup orientation measurement [22].

Considering the case of an obese patient, the measurement accuracy could be drastically impaired. Hence, the pointer-based palpation has been ascertained by many groups [23]-[27] as the major technical limitation of THA navigation systems. This prevalent draw back together with the current trend towards minimally invasive surgeries lead to the approach of replacing the pointer-based digitization procedure by CT scan imaging [28]-[31]. Due to its depth penetration property CT scan imaging, is more appropriate to locate bony structures covered by subcutaneous tissues. Moreover CT scanning does not expose the patient or the medical staff to radiation. However, the integration of CT scanning into the work flow of a navigated orthopaedics surgery gives rise to new challenges. Beyond the general issues of introducing CT scan imaging to THA inter versions, the processing of the CT scan images are subject to many different types of artifacts, degrading the quality of the data [32].In order to extract the APP based on CT scan images, sophisticated algorithms have to be applied, the higher the error in localizing the correct depth of the bone. A possible solutions to compensate for this kind of error has been presented by Barratt et al[41].Within a self calibration scheme, US-derived surface points of a pelvis were registered to the corresponding preoperative CT scan of the same bone during regid registration, not only 3-D transformation but also the calibration matrix, describing the position and orientation of the US image with respect to the localizer attached to the US-Probe, is optimized the initial 3-D transformation was there by established by simulated digitized points. We developed a new method for the intra operative determination of APP based on CT scan derived point clouds. In contrast to the self 
calibration approach [41], no preoperative data of the respective bone are available. Instead, different SSM's of the pelvis are used to extra operative the sparse CT data.

Therefore a nonregid registration problem has to be solved together with the optimization of the calibration matrix. The problem definition is in some cases the patients with the saviour damage of a pelvic bone a reported which takes lots of time and expenditure to resolve it technologies like digital image processing will help amount of damage cost by verifying CT scanned images the measurement utility which has been adopted will give the conclude results. Which will help the expert people to give their opinion based a statistical value generated. Present method is to register intra operative US images to the preoperative CT scan of the same patient. An intensity-based method was used in both cases to estimate the optimal rigid registration between both COS. Therefore, the APP could be easily defined in the CT-COS and transferred to the intra operative COS based on the estimated transformation. And proposed method is an accurate measurement utility by image calibration method for estimating pelvic bone orientation. Using the standard set of measurement utilities for length, orientation and diameter estimation involvement of in the system to give the solution or conclusion. The experts' advice can be made from the local or remote position to assist treatment process the image given as input will be transform by calibration and related process will help an extensive the important statistics values.

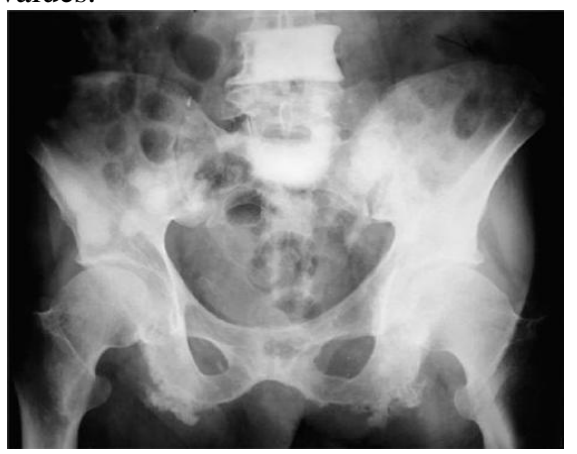

Fig.1 Proper image

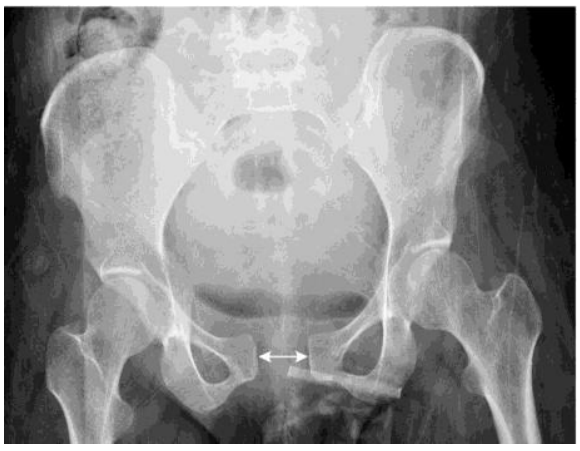

Fig.2 Improper image

\section{Related Work}

Image-based hip navigation preclinical evaluation of a CT-free image-guided surgical Navigation system for acetabular cup placement, the system was introduced into clinical routine. The Computation of the angular orientation of the cup is based on reference coordinates from the anterior pelvic plane (APP) concept. A hybrid strategy for pelvic landmark acquisition has been introduced involving percutaneous pointer-based digitization with the noninvasive biplanar landmark reconstruction using multiple registered fluoroscopy images.

A kinematic model for calculating cup alignment error during total hip arthroplasty on. Reduced range of motion, prosthetic impingement, and joint dislocation can all result from misalignment of the acetabular component (i.e. cup alignment) in patients undergoing total hip arthroplasty. To achieve cup alignment accuracyon the order of 11, even a minor failure to correctlyidenti fythe anatomical landmarks can lead to much higher inaccuracies in the final cup alignment. This main contribution of the study is to derive a closed form mathematical solution for the analysis of the effect of inaccuracies related to the determination of the location of the APP landmarks and their effect on the final cup alignment during total hip arthroplasty.

Automatic fluoroscopic Image Calibration for Traumatology Intervention Guidance an applied to a common operation in traumatology: the distal targeting during intra-medullary nailing. We will also show how calibrated data from fluoroscopy imaging system combined to virtual reality techniques can guide the surgeon in such difficult tasks. The 3D positions of the holes have been measured with the tracking system and compared with these ones of the 3D reconstructed model of the nail. The maximum errors are about $2 \mathrm{~mm}$ for the centre position and $2^{\circ}$ for the axis orientation. In the future, a robotic arm placing the drilling motor in proper position under electronic guidance by the surgeon can be envisaged.

Accuracy of Computer Navigation for Acetabular Component Placement in THA the accuracy and precision of any computer aided surgical device is critical to its utility. We asked the following question: how accurate and precise are the values measured by an imageless computer navigation system as compared with those measured using postoperative CT scans? Twenty-five patients ( 25 hips) underwent primary THA using an imageless computer navigation system for placement of the acetabular component. Inclination and ante version were measured in the operative coordinate system.

A comparison study assessing the feasibility of ultrasound initialized deformable bone models, on earlier to combine several automatic methods for statistical bone shape prediction and ultrasound segmentation and calibration to provide the intended rapid and accurate visualization. They compared the use of a tracked 
digitizing pointer and ultrasound for acquiring landmarks and bone surface points for the estimation of two cast proximal femurs.

Active Shape Models-Their Training and Application on a method for building models by learning patterns of variability from training set of correctly annotated images. These models can be used for image search in an iterative refinement algorithm analogous to that employed by Active Contour Models (Snakes). The key difference is that our Active Shape Models can only deform to fit the data in ways consistent with the training set. We show several practical examples where we have built such models and used them to locate partially occluded objects in noisy, cluttered images.

Normal Probability Plots and Tests for Normal probability plots are often used as an informal means of assessing the non normality of a set of data. One problem confronting persons inexperienced with probability plots is that considerable practice is necessary before one can learn to judge them with any degree of confidence. Some objective measure of the straightness of a probability plot would be helpful, especially for students just beginning their statistical education.

Statistical deformable bone models for robust 3D surface extrapolation from sparse data, proposed technology brings a variety of advantages to orthopaedic and other surgical procedures, such asimproved accuracy and safety, often reduced radiation exposure, and improved surgical reality through 3D visualization. In particular navigation based on shape deformation opens the door to more minimally invasive approaches. Future work will include the expansion of our training set of bone shapes to build more comprehensive shape models. We will also target other anatomies like the distal femur, the entire femur and the spine. Additionally, the order and the location of the surface points that are provided to the shape deformation algorithm might have an influence on the overall estimation result.

Reducing the risk of dislocation after total hip arthroplasty, compared with those in a control group of Malposition of the acetabular component is a risk factor for post-operative dislocation after Total hip replacement (THR). We have investigated the influence of the orientation of the acetabular component on the probability of dislocation.

\section{System Design}

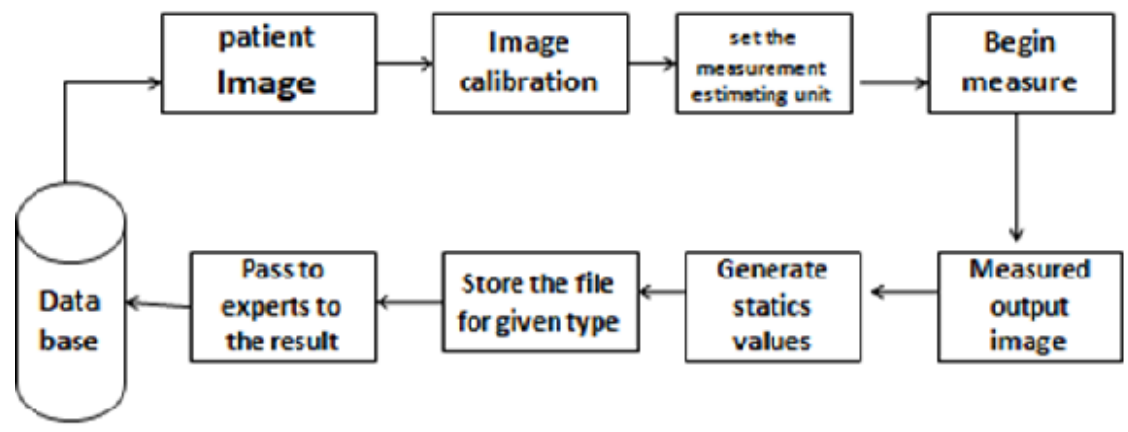

Fig.3 Proposed system design

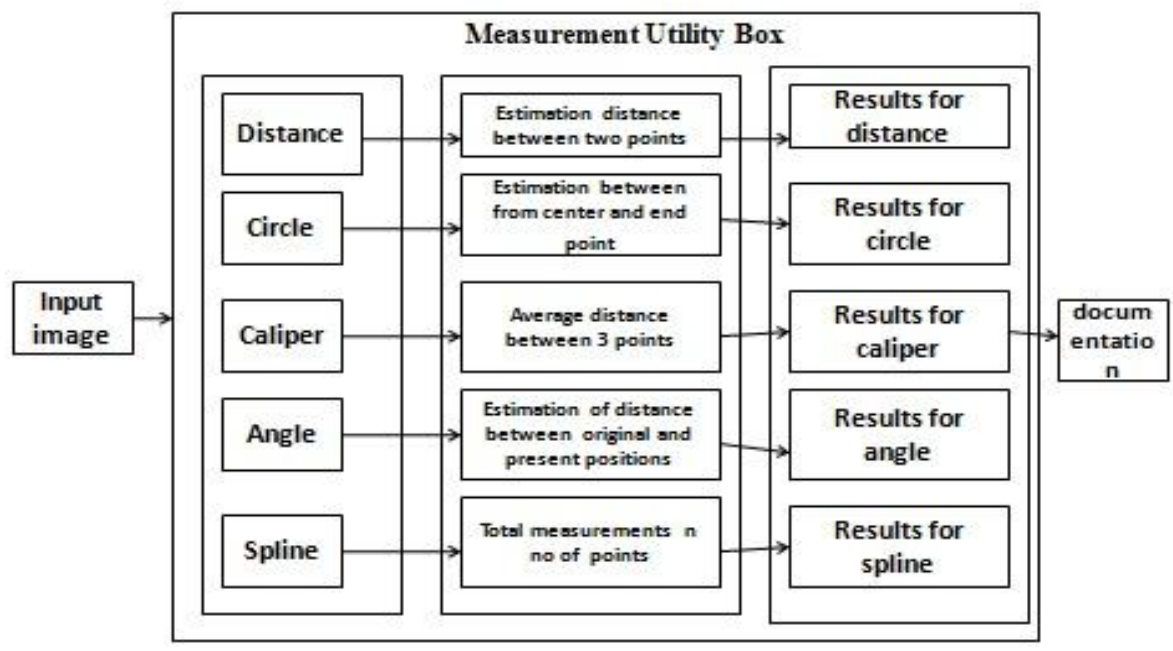

Fig.4 System architecture 
Above system design shows that the particulars of the patient are stored in Database (DB). The image of the anterior pelvic plane will be passed to the image calibration from Database. Given pixels $p, q$ and $z$ with coordinates $(x, y),(s, t),(u, v)$ respectively, the distance Function D has following properties:

$$
\begin{aligned}
& \text { a. } D(p, q) 0[\mathrm{D}(\mathrm{p}, \mathrm{q})=0 \text {, if } \mathrm{p}=\mathrm{q} \\
& \text { b. } D(p, q)=D(q, p) \\
& \text { c. } D(p, z) D(p, q)+D(q, z)
\end{aligned}
$$

A calibrate array is mechanically quite stable, the pixels have a fixed geometric relationship. Each pixel within the array however has unique light sensitivity characteristics. Because these characteristics affect camera performance, they must be removed through calibration. The process by which a calibrate image is calibrated is known as flat fielding or shading correction.

\subsection{Flat fielding}

Flat fielding requires the acquisition of two calibration images. Both images must be obtained close to the time of the object exposures to be calibrated, because these images are used to provide quantitative calibration of pixel signals. If you are going to compare corrected images, all must be corrected using the same calibration images.

3.2 Procedure

First, a bias or dark image is acquired. In a bias image, all of the pixels have approximately the same value, which consists of the electronic offset of the system and any inherent structure of the calibrate. A dark image contains the dark signal as well as the standard bias component. A dark image is most useful for correcting long exposures with low light levels. The flat field image measures the response of each pixel in the calibrate array to illumination. The optical system introduces some variation in response. The reason being the flat fielding process corrects for uneven illumination only if that illumination is a stable characteristic of each object exposure. The flat field image should be as representative of the background illumination as possible.

The illumination should be bright enough or the exposure long enough, that the calibrate pixel signals are at least 25 percent of full scale. When a raw image is created the flat fielding technique can be applied. The flat field image is corrected for electronic offset by subtracting the bias/dark image from it. The mean pixel value of the resulting corrected flat field image is ascertained. The mean pixel value is unique to each combination of bias/dark and flat field image. The bias/dark image is then subtracted from the exposed image.

The resulting image is multiplied by the mean pixel value of the corrected flat field image and finally divided by the corrected flat field image itself. (If the corrected flat field image contains a pixel with zero value, that pixel in the corrected image is assigned a full scale value.) The mean pixel value of the corrected image is not exactly the same as that of the raw image. However, multiplying by the mean pixel value of the corrected flat field image keeps the number more or less the same. Calibration means dimension of the image i.e., the length, breadth and depth are measured. The measurements are estimated in units. From there this will be passed for setting the measurements for estimation of units and then measurement output image will come this generates statistical values of the image.

\subsection{Length}

A path from pixel $\mathrm{p}$ with coordinates $(x, y)$ to pixel $q$ with coordinates $(s, t)$ is a sequence of distinct pixels with Coordinates : $\left(x_{0}, y_{0}\right),\left(x_{1}, y_{1}\right),\left(x_{2}, y_{2}\right) \ldots\left(x_{n}, y_{n}\right)$, where $\left(x_{0}, y_{0}\right)=(x, y)$ and $\left(x_{n}, y_{n}\right)=(s, t) ;\left(x_{i}, y_{i}\right)$ is adjacent to $\left(x_{i-l}, y_{i-1}\right)$ here $n$ is the length of the path. Given pixels $p, q$ and $z$ with coordinates $(x, y),(s, t),(u, v)$ respectively, the distance function $\mathrm{D}$ has following properties:
a. $D(p, q) O[\mathrm{D}(\mathrm{p}, \mathrm{q})=0$, if $\mathrm{p}=\mathrm{q}]$
b. $D(p, q)=D(q, p)$
c. $D(p, z) D(p, q)+D(q, z)$
3.4Diameter

A circle is described by $(x-x c)^{\wedge} 2+(y-y c)^{\wedge} 2=r^{\wedge} 2$. Where $(x, y)$ is on the circle, $(x c, y c)$ is the center of the circle and $\mathrm{r}$ is its radius.

\subsection{Orientation}

Angle between Two Points on a Sphere. The angle that two vectors pointing to different locations on the surface of a sphere make with each other is given in terms of the latitude and longitude of those points $\cos (\theta)=\sin (\theta 1) \sin (\theta 2)+\cos (\theta 1) \cos (\theta 2) \cos (\theta 1-\theta 2)$

Where $i$ is the latitude for each point with $i=1,2$ and $i$ is the longitudes for each point with $i=1,2$ and is the angle between the two points. Although the above equation is good for any pair of points on the sphere, the numerical precision required for an accurate answer when the angle is small becomes prohibitive. In the case that the two where $\sin \theta$ and $\cos \theta$ are the change in those coordinates between the two points 2 , these will be stored and documentation of the values will be filed. When these values are required will be passed to the 
experts or the Doctors or to the persons who are in need of these values for examination and proper treatment. Again this information will be passed to database for storage.

\section{Algorithm}

Step1: Initially passes input images to the system

$$
\text { Input=imread (pat1.jpg) }
$$

Step2: Perform the calibration process to make such that images were calibrated and get the

Next

Process.

Step3: Set the variables for the beginning of measurement.

Step4: Begin measurement

Set point $\mathrm{A}$ and $\mathrm{B}$ on longitudes and measure the distance between them.

For measurement of radius take a point on circumference and the distance between the center gives radius. (Diameter $=2$ Xradius)

Set points $\mathrm{A}, \mathrm{B}$ and $\mathrm{C}$ on the sphere and the average distance between three points gives the Measurement of caliper.

$$
C=\frac{\mathrm{A}+\mathrm{B}}{\mathrm{x}}
$$

Where' $\mathrm{x}$ ' is a positioning allignment

To measure the angle set points $\mathrm{A}, \mathrm{B} \& \mathrm{C}$, and the distance between two segments formed by these Points.

In case of spline to find total measurement.

$$
L=\sum_{i=1}^{n}\left(x_{i}+x_{i+1}\right)
$$

Where ' $x$ ' is number of location points

End measurement

\section{Materials And Methods}

The work requires the images of the pelvic bone which are taken from the standard resources. With the aid of the Matlab-8 and Digital Image Processing methods the work will give us the accurate result; it indeed referred with an expert person before concluding.

\section{Experimental Results}

The proposed technique for estimating the orientation of the pelvic bone will give rise to the new trend to the medical science .In this work the basic and important geometrical features of a pelvic bone are estimated and releasing statistical values which are used for the reorganization and purpose the disease patient. The system uses expert's suggestions to understand the impact of displacement of the pelvic bone.

Table I data file created by patient 1 measurement tool

\begin{tabular}{|c|l|l|l|l|l|l|l|}
\hline $\begin{array}{c}\text { Image size after } \\
\text { calibration }\end{array}$ & Num & Type & Unit & Values & Intensity & Xcoords & Ycoords \\
\hline & & & & & & & \\
$530 X 750 \times 1$ & 1 & Distance & Pixel & 6.740200 & 2.042075 & 3.31 & 3.99 \\
& 2 & Circle(R) & Pixel & 1.260595 & 1.809656 & 3.56 & 2.54 \\
& 3 & Caliper & Pixel & 7.887933 & 2.190293 & 1.83 & 5.37 \\
& 4 & Angle & Deg & 1.188080 & 9.605632 & 4.84 & 2.74 \\
& 5 & Spline & Pixel & 5.393994 & 1.110684 & 8.58 & 6.57 \\
\hline
\end{tabular}

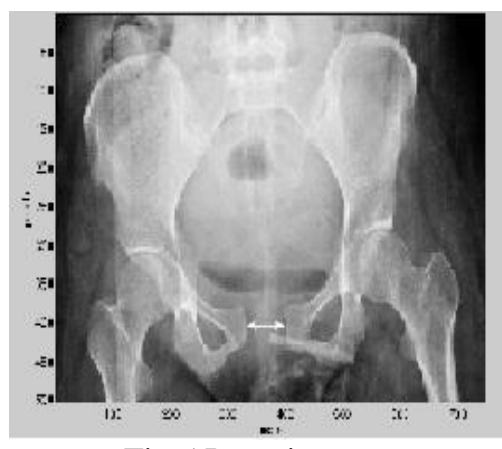

Fig.5 Input image

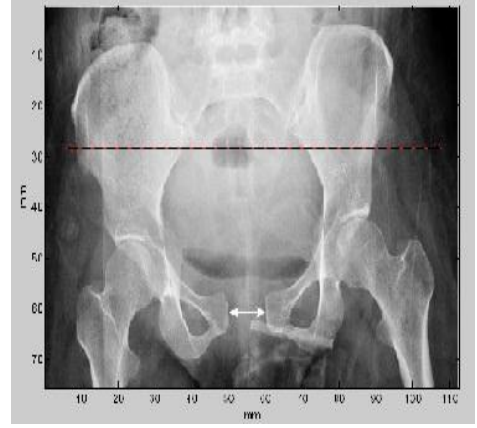

Fig.6 Calibration result 


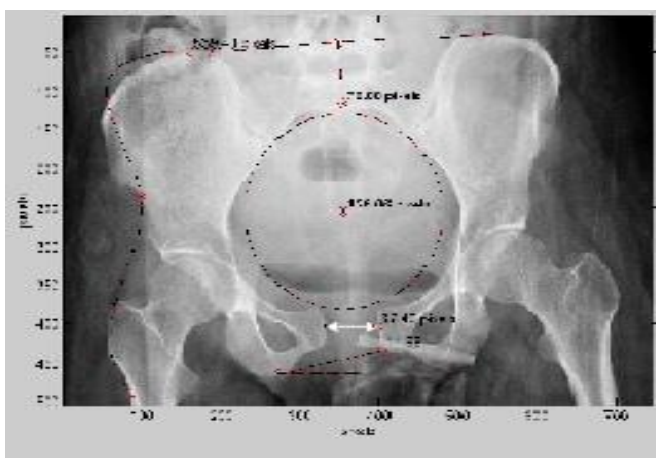

Fig.7 Output image

Data $\mathrm{t1}$ le created by measuretool.m

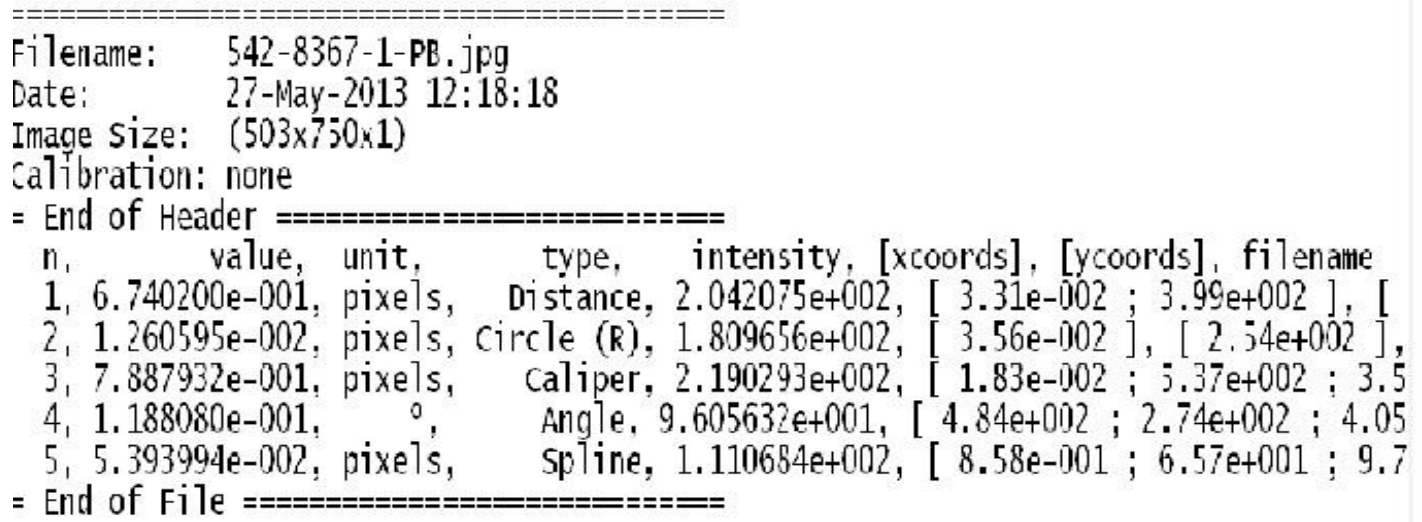

Fig.8 Resulting stats of output image

\section{Conclusion}

In this paper a novel approach is used to determine the ORIENTATION OF PELVIC BONE from CT scan images of the pelvis. A hierarchical registration scheme based on different SSM's of the pelvis has been developed to compensate for variations of measurement utility tools by image calibration in different soft tissues. The proposed method requires only CT scan images of the conventional APP regions to determine the orientation of pelvis. The additional effort of CT integration into the clinical root of computer assisted orthopedic surgeries and involvement of the experts to give the solutions to the patients. The core advantage of the system can be operated from remote location from experts and other advantage is taking minimum time and less expensive compared with the existing system. The presented approach has the potential to replace the invasive pointer-based registration by acquisition of sparse, non invasive CT images.

\section{References}

[1] D. McCollum and W. Gray, "Dislocation after total hip arthroplasty: Causes and prevention,” Clin. Orthop. Relat. Res., vol. 261, 1990.

[2] B. Jaramaz, C. Nikou, and A. DiGioia, "Effect of cup orientation and neck length in range of motion simulation," in Proc. 43rd Annu. Meeting Orthopaedic Res. Soc., 1997, vol. 22, p. 286.

[3] L. Dorr and Z. Wan, "Causes of and treatment protocol for instability of total hip replacement," Clin. Orthop. Relat. Res., vol. 355, pp. 144-151, 1998.

[4] J. Kennedy, W. Rogers, K. Soff, R. Sullivan, D. Griffen, and L. Sheehan, "Effect of acetabular component orientation on recurrent dislocation, pelvic osteolysis, polyethylene wear, and component migration," J.Arthroplasty, vol. 5, pp. 530-534, 1998.

[5] G. Lewinnek, J. Lewis, R. Tarr, C. Compere, and J. Zimmerman, "Dislocation after total hip replacement arthroplasties," J. Bone Joint Surg. Amer., vol. 60, pp. 217-220, 1978.

[6] D. Murray, "The definition and measurement of acetabular orientation," J. Bone Joint Surg. Br., vol. 75, pp. 228-232, 1993.

[7] R. Biedermann, A. Tonin, M. Krismer, F. Rachbauer, G. Eibl, and B. Stoeckl, "Reducing the risk of dislocation after total hip arthroplasty," J. Bone Joint Surg. Br., vol. 87, pp. 762-769, 2005.

[8] S. Steppacher, J.Kowal, and S.Murphy, "Improving cup positioning using a mechanical navigation instrument," Clin. Orthop. Relat. Res., vol. 469, pp. 423-428, 2010.

[9] G. Zheng, S. Echeverri, W. Xie, an d L. Nolte, "PS-GANS: A patientspecific, gravity assisted navigation system for acetabular cup placement," in Lecture Notes in Computer Science. vol. 6689, Berlin, Germany:Springer, 2011, pp. 101-112.

[10] A. DiGioia, B. Jaramaz, M. Blackwell, D. Simon, F. Morgan, J. Moody, C. Nikou, B. Colgan, C. Aston, R. Labarca, E. Kischell, and T. Kanade, "Image guided navigation system to measure intraoperatively acetabular implant alignment," Clin. Orthop. Relat. Res., vol. 355, pp. 8-22, 1998.

[11] K. Widmer and P. Gruetzner, "Joint replacement-Total hip replacement with CT-based navigation,” Injury, vol. 35, pp. 84-89, 2004. 
[12] R. Hofstetter, M. Slomczykowski, M. Sati, and L. Nolte, "Fluoroscopy as an imaging means for computer-assisted surgical navigation," Comput. Aid Surg., vol. 4, pp. 65-76, 1999.

[13] G. Zheng, A. Marx, U. Langlotz, K. Widmer, M. Buttaro, and L. Nolte, "A hybrid CT-free navigation system for total hip arthroplasty," Comput. Aid Surg., vol. 7, pp. 129-145, 2002.

[14] M. Nogler, O. Kessler, A. Prassl, B. Donnelly, R. Streicher, J. Sledge, and M. Krismer, "Reduced variability of acetabular cup positioning with use of an imageless navigation system," Clin. Orthop. Relat. Res., vol. 426,pp. 159-163, 2004.

[15] T. Kalteis, M. Handel, H. Baethis, L. Perlick, M. Tingart, and J. Grifka, "Imageless navigation for insertion of the acetabular component in total hip arthroplasty,” J. Bone Joint Surg. Br., vol. 88, pp. 163-167, 2005.

[16] J. Spencer, R. Day, K. Sloan, and R. Beaver, "Computer navigation of the acetabular component," J. Bone Joint Surg. Br., vol. 88B, pp. 972-975, 2006.

[17] J. Ryan, A. Jamali, and W. Bargar, "Accuracy of computer navigation for acetabular component placement in THA," Clin. Orthop. Relat. Res., vol. 468, pp. 169-177, 2010.

[18] T. Leenders, D. Vandevelde, G. Mahieu, and R. Nuyts, "Reduction in variability of acetabular cup abduction using computer assisted surgery: A prospective and randomized study," Comput. Aid Surg., vol. 7, pp. 99-106, 2002.

[19] B. Jolles, P.Genoud, and P.Hoffmeyer, "Computer-assisted cup placement techniques in total hip arthroplasty improve accuracy of placement," Clin. Orthop. Relat. Res., vol. 426, pp. 174-179, 2004.

[20] B. Jaramaz, A. DiGioia, M. Blackwell, and D. Nolan, "Computer assisted measurement of cup placement in total hip replacement," Clin. Orthop. Relat. Res., vol. 354, pp. 70-80, 1998.

[21] M. Tannast, U. Langlotz, K. Siebenrock, M. Wiese, K. Bernsmann, and F. Langlotz, "Anatomic referencing of cup orientation in total hip arthroplasty," Clin. Orthop. Relat. Res., vol. 436, pp. 144-150, 2005.

[22] A. Wolf, A. DiGioia, A. Mor, and B. Jaramaz, "A kinematic model for calculating cup alignment error during total hip arthroplasty,” J. Biomech., vol. 38, pp. 2257-2265, 2005.

[23] J. Richolt, H. Effenberger, and M. Rittmeister, "How does soft tissue distribution affect anteversion accuracy of the palpation procedure in imagefree acetabular cup navigation? An ultrasonic assessment," Comput. Aid Surg., vol. 10, pp. 87-92, 2005.

[24] S. Parratte and J. Argenson, "Validation and usefulness of a computerassisted cup-positioning system in total hip arthroplasty," J. Bone Joint Surg. Amer., vol. 89, pp. 494-499, 2007.

[25] H. Kiefer and A. Othman, "Ultrasound vs. pointer palpation based method in THA navigation. a comparative study," Orthopedics, vol. 10 (Suppl), pp. 153-156, 2007, 2012.

[26] Y. Lee and T. Yoon, "Error in acetabular socket alignment due to the thick anterior pelvic soft tissues," J. Arthroplasty, vol. 23, pp. 699-706, 2008.

[27] S. Tsukada and M. Wakui, "Decreased accuracy of acetabular cup placement for imageless navigation in obese patients," J. Orthop. Sci., vol. 15, pp. 758-763, 2010.

[28] C. Chan, D. Barratt, P. Edwards, G. Penney, M. Slomczykowski, T. Carter, and D. Hawkes, "Cadaver validation of the use of ultrasound for $3 \mathrm{dmodel}$ instantiation of bony anatomy in image guided orthopaedic surgery,"in Lecture Notes in Computer Science, vol. 3217, Berlin, Germany: Springer, 2004, pp. 397-404.

[29] P. Foroughi, D. Song, G. Chintalapani, R. Taylor, and G. Fichtinger, "Localization of pelvic anatomical coordinate system using us/atlas registration for total hip replacement," in Lecture Notes in Computer Science.vol. 5242, Berlin, Germany: Springer, 2008, pp. 871-879.

[30] D. Barratt, C. Chan, P. Edwards, G. Penney, M. Slomczykowski, T. Carter, and D. Hawkes, "Instantiation and registration of statistical shape models of the femur and pelvis using 3d ultrasound imaging," Med. Image Anal.,vol. 12, pp. 358-374, 2008.

[31] S. Parratte, P. Kilian, P. Pauly, P. Champsaur, and J. Argenson, "The use of ultrasound in acquisition of the anterior pelvic plane in computer-assisted total hip replacement," J. Bone Joint Surg. Br., vol. 90-B, pp. 258-263,2008.

[32] P. Hoskins, M. Kevin, and T. Abigail, Diagnostic Ultrasound: Physics and Equipment. Cambridge, U.K.: Cambridge Univ. Press, 2010.

[33] D. Amin, T. Kanade, A. DiGioia, and B. Jaramaz, "Ultrasound registration of the bone surface for surgical navigation," Comput. Aid Surg., vol. 8, pp. 1-15, 2003.

[34] G. Penney, D. Barratt, C. Chan,M. Slomczykowski, T. Carter, P. Edwards, and D. Hawkes, "Cadaver validation of intensity-based ultrasound to CT registration," Med. Image Anal., vol. 10, pp. 385-395, 2006.

[35] G. Dardenne, S. Dusseau, C. Hamitouche, C. Lefevre, and E. Stindel, "Toward a dynamic approach of THA planning based on ultrasound," Clin. Orthop. Relat. Res., vol. 467, pp. 901-908, 2009.

[36] S. Ghanavati, P. Mousavi, G. Fichtinger, P. Foroughi, and P. Abolmaesumi, "Multi-slice to volume registration of ultrasound data to a statistical atlas of human pelvis," in SPIE Medical Imaging, vol. 7625. Ottawa, Canada: SPIE Digital Library, 2010 , pp. 76 250O-1-76 250O-10

[37] P. Detmer, G. Bashein, T. Hodges, K. Beach, E. Filer, D. Burns, and D. Strandness, "3D ultrasonic image feature localization based on magnetic scanhead tracking: In vitro calibration and validation," Ultrasound Med. Biol., vol. 20, pp. 923-936, 1994

[38] R. Prager, R. Rohling, A. Gee, and L. Berman, "Rapid calibration for 3-D freehand ultrasound," Ultrasound Med. Biol., vol. 24, pp. 855-869, 1998 .

[39] J. Kowal, C. Amstutz, M. Caversaccio, and L. Nolte, "On the development and comparative evaluation of an ultrasound b-mode probe calibration method," Comput. Aided Surg., vol. 8, pp. 107-119, 2003

[40] S. Goss, R. Johnston, and F. Dunn, "Comprehensive compilation of empirical ultrasonic properties of mammalian tissues," J. Acoust. Soc. Amer., vol. 64, pp. 423-457, 1978.

[41] D. Barratt, G. Penney, C. Chan,M. Slomczykowski, T. Carter, P. Edwards, and D. Hawkes, "Self-calibrating 3D-ultrasound-based bone registration for minimally invasive orthopedic surgery,” IEEE Trans. Med. Imag.,vol. 25, no. 3, pp. 312-323, Mar. 2006. 